\title{
Stochastic Processes
}

- A stochastic process

$$
X=\{X(t)\}
$$

is a time series of random variables.

- $X(t)$ (or $X_{t}$ ) is a random variable for each time $t$ and is usually called the state of the process at time $t$.

- A realization of $X$ is called a sample path.

- A sample path defines an ordinary function of $t$.

Of all the intellectual hurdles which the human mind has confronted and has overcome in the last fifteen hundred years, the one which seems to me to have been the most amazing in character and the most stupendous in the scope of its consequences is the one relating to the problem of motion. — Herbert Butterfield (1900-1979)

\section{Stochastic Processes (concluded)}

- If the times $t$ form a countable set, $X$ is called a discrete-time stochastic process or a time series.

- In this case, subscripts rather than parentheses are usually employed, as in

$$
X=\left\{X_{n}\right\}
$$

- If the times form a continuum, $X$ is called a continuous-time stochastic process. 


\section{Random Walks}

- The binomial model is a random walk in disguise.

- Consider a particle on the integer line, $0, \pm 1, \pm 2, \ldots$.

- In each time step, it can make one move to the right with probability $p$ or one move to the left with probability $1-p$.

- This random walk is symmetric when $p=1 / 2$.

- Connection with the BOPM: The particle's position denotes the cumulative number of up moves minus that of down moves.

\section{Random Walk with Drift}

$$
X_{n}=\mu+X_{n-1}+\xi_{n}
$$

- $\xi_{n}$ are independent and identically distributed with zero mean.

- Drift $\mu$ is the expected change per period.

- Note that this process is continuous in space.

\section{Martingales $^{\mathrm{a}}$}

- $\{X(t), t \geq 0\}$ is a martingale if $E[|X(t)|]<\infty$ for $t \geq 0$ and

$$
E[X(t) \mid X(u), 0 \leq u \leq s]=X(s), \quad s \leq t
$$

- In the discrete-time setting, a martingale means

$$
E\left[X_{n+1} \mid X_{1}, X_{2}, \ldots, X_{n}\right]=X_{n} .
$$

- $X_{n}$ can be interpreted as a gambler's fortune after the $n$th gamble.

- Identity (40) then says the expected fortune after the $(n+1)$ th gamble equals the fortune after the $n$th gamble regardless of what may have occurred before.

${ }^{a}$ The origin of the name is somewhat obscure. 


\section{Martingales (concluded)}

- A martingale is therefore a notion of fair games.

- Apply the law of iterated conditional expectations to both sides of Eq. (40) on p. 403 to yield

$$
E\left[X_{n}\right]=E\left[X_{1}\right]
$$

for all $n$.

- Similarly, $E[X(t)]=E[X(0)]$ in the continuous-time case.

\section{Still a Martingale? (continued)}

- Well, no.

- Consider this random walk with drift:

$$
X_{i}= \begin{cases}X_{i-1}+\xi_{i}, & \text { if } i \text { is even, } \\ X_{i-2}, & \text { otherwise. }\end{cases}
$$

- Above, $\xi_{n}$ are random variables with zero mean.

${ }^{\mathrm{a} C o n t r i b u t e d ~ b y ~ M r . ~ Z h a n g, ~ A n n-S h e n g ~(B 89201033) ~ o n ~ A p r i l ~ 13, ~}$ 2005

\section{Still a Martingale? (concluded)}

- It is not hard to see that

$$
E\left[X_{i} \mid X_{i-1}\right]= \begin{cases}X_{i-1}, & \text { if } i \text { is even } \\ X_{i-1}, & \text { otherwise. }\end{cases}
$$

- Hence it is a martingale by the "new" definition.

- But

$$
E\left[X_{i} \mid \ldots, X_{i-2}, X_{i-1}\right]= \begin{cases}X_{i-1}, & \text { if } i \text { is even } \\ X_{i-2}, & \text { otherwise }\end{cases}
$$

- Hence it is not a martingale by the original definition. 


\section{Example}

- Consider the stochastic process

$$
\left\{Z_{n} \equiv \sum_{i=1}^{n} X_{i}, n \geq 1\right\}
$$

where $X_{i}$ are independent random variables with zero mean.

- This process is a martingale because

$$
\begin{aligned}
& E\left[Z_{n+1} \mid Z_{1}, Z_{2}, \ldots, Z_{n}\right] \\
= & E\left[Z_{n}+X_{n+1} \mid Z_{1}, Z_{2}, \ldots, Z_{n}\right] \\
= & E\left[Z_{n} \mid Z_{1}, Z_{2}, \ldots, Z_{n}\right]+E\left[X_{n+1} \mid Z_{1}, Z_{2}, \ldots, Z_{n}\right] \\
= & Z_{n}+E\left[X_{n+1}\right]=Z_{n} .
\end{aligned}
$$

\section{Probability Measure (continued)}

- A stochastic process $\{X(t), t \geq 0\}$ is a martingale with respect to information sets $\left\{I_{t}\right\}$ if, for all $t \geq 0$, $E[|X(t)|]<\infty$ and

$$
E\left[X(u) \mid I_{t}\right]=X(t)
$$

for all $u>t$

- The discrete-time version: For all $n>0$,

$$
E\left[X_{n+1} \mid I_{n}\right]=X_{n},
$$

given the information sets $\left\{I_{n}\right\}$.

\section{Probability Measure}

- A martingale is defined with respect to a probability measure, under which the expectation is taken.

- A probability measure assigns probabilities to states of the world.

- A martingale is also defined with respect to an information set.

- In the characterizations (39)-(40) on p. 403, the information set contains the current and past values of $X$ by default.

- But it needs not be so.

\section{Probability Measure (concluded)}

- The above implies $E\left[X_{n+m} \mid I_{n}\right]=X_{n}$ for any $m>0$ by Eq. (15) on p. 137.

- A typical $I_{n}$ is the price information up to time $n$.

- Then the above identity says the FVs of $X$ will not deviate systematically from today's value given the price history. 


\section{Example}

- Consider the stochastic process $\left\{Z_{n}-n \mu, n \geq 1\right\}$.

$-Z_{n} \equiv \sum_{i=1}^{n} X_{i}$.

- $X_{1}, X_{2}, \ldots$ are independent random variables with mean $\mu$.

- Now,

$$
\begin{aligned}
& E\left[Z_{n+1}-(n+1) \mu \mid X_{1}, X_{2}, \ldots, X_{n}\right] \\
= & E\left[Z_{n+1} \mid X_{1}, X_{2}, \ldots, X_{n}\right]-(n+1) \mu \\
= & Z_{n}+\mu-(n+1) \mu \\
= & Z_{n}-n \mu .
\end{aligned}
$$

\section{Martingale Pricing}

- Recall that the price of a European option is the expected discounted future payoff at expiration in a risk-neutral economy.

- This principle can be generalized using the concept of martingale.

- Recall the recursive valuation of European option via

$$
C=\left[p C_{u}+(1-p) C_{d}\right] / R .
$$

$-p$ is the risk-neutral probability.

$-\$ 1$ grows to $\$ R$ in a period.

\section{Example (concluded)}

- Define

$$
I_{n} \equiv\left\{X_{1}, X_{2}, \ldots, X_{n}\right\}
$$

- Then

$$
\left\{Z_{n}-n \mu, n \geq 1\right\}
$$

\section{Martingale Pricing (continued)}

- Let $C(i)$ denote the value of the option at time $i$.

- Consider the discount process

$$
\left\{C(i) / R^{i}, i=0,1, \ldots, n\right\} .
$$

- Then,

$$
E\left[\frac{C(i+1)}{R^{i+1}} \mid C(i)=C\right]=\frac{p C_{u}+(1-p) C_{d}}{R^{i+1}}=\frac{C}{R^{i}} .
$$




\section{Martingale Pricing (continued)}

- It is easy to show that

$$
E\left[\frac{C(k)}{R^{k}} \mid C(i)=C\right]=\frac{C}{R^{i}}, \quad i \leq k .
$$

- This formulation assumes: ${ }^{\mathrm{a}}$

1. The model is Markovian in that the distribution of the future is determined by the present (time $i$ ) and not the past.

2. The payoff depends only on the terminal price of the underlying asset (Asian options do not qualify).

${ }^{a}$ Contributed by Mr. Wang, Liang-Kai (Ph.D. student, ECE, University of Wisconsin-Madison) and Mr. Hsiao, Huan-Wen (B90902081) on May 3, 2006.

\section{Martingale Pricing (continued)}

- Equation (43) holds for all assets, not just options.

- When interest rates are stochastic, the equation becomes

$$
\frac{C(i)}{M(i)}=E_{i}^{\pi}\left[\frac{C(k)}{M(k)}\right], \quad i \leq k .
$$

- $M(j)$ is the balance in the money market account at time $j$ using the rollover strategy with an initial investment of $\$ 1$.

- So it is called the bank account process.

- It says the discount process is a martingale under $\pi$.

\section{Martingale Pricing (continued)}

- In general, the discount process is a martingale in that

$$
E_{i}^{\pi}\left[\frac{C(k)}{R^{k}}\right]=\frac{C(i)}{R^{i}}, \quad i \leq k .
$$

- $E_{i}^{\pi}$ is taken under the risk-neutral probability conditional on the price information up to time $i$.

- This risk-neutral probability is also called the EMM, or the equivalent martingale (probability) measure.

\section{Martingale Pricing (concluded)}

- If interest rates are stochastic, then $M(j)$ is a random variable.

$-M(0)=1$.

$-M(j)$ is known at time $j-1$.

- Identity (44) on p. 418 is the general formulation of risk-neutral valuation.

Theorem 14 A discrete-time model is arbitrage-free if and only if there exists a probability measure such that the discount process is a martingale. This probability measure is called the risk-neutral probability measure. 


\section{Futures Price under the BOPM}

- Futures prices form a martingale under the risk-neutral probability.

- The expected futures price in the next period is

$$
p_{\mathrm{f}} F u+\left(1-p_{\mathrm{f}}\right) F d=F\left(\frac{1-d}{u-d} u+\frac{u-1}{u-d} d\right)=F
$$

(p. 380).

- Can be generalized to

$$
F_{i}=E_{i}^{\pi}\left[F_{k}\right], \quad i \leq k
$$

where $F_{i}$ is the futures price at time $i$.

- It holds under stochastic interest rates.

\section{Martingale Pricing and Numeraire (concluded)}

- Choose $S$ as numeraire.

- Martingale pricing says there exists a risk-neutral probability $\pi$ under which the relative price of any asset $C$ is a martingale:

$$
\frac{C(i)}{S(i)}=E_{i}^{\pi}\left[\frac{C(k)}{S(k)}\right], \quad i \leq k .
$$

- $S(j)$ denotes the price of $S$ at time $j$.

- So the discount process remains a martingale.

\section{Example}

- Take the binomial model with two assets.

- In a period, asset one's price can go from $S$ to $S_{1}$ or $S_{2}$.

- In a period, asset two's price can go from $P$ to $P_{1}$ or $P_{2}$.

- Assume

$$
\frac{S_{1}}{P_{1}}<\frac{S}{P}<\frac{S_{2}}{P_{2}}
$$

to rule out arbitrage opportunities.

\footnotetext{
a Leon Walras (1834-1910).
} 


\section{Example (continued)}

- For any derivative security, let $C_{1}$ be its price at time one if asset one's price moves to $S_{1}$.

- Let $C_{2}$ be its price at time one if asset one's price moves to $S_{2}$

- Replicate the derivative by solving

$$
\begin{aligned}
& \alpha S_{1}+\beta P_{1}=C_{1}, \\
& \alpha S_{2}+\beta P_{2}=C_{2},
\end{aligned}
$$

using $\alpha$ units of asset one and $\beta$ units of asset two.

\section{Example (concluded)}

- It is easy to verify that

$$
\frac{C}{P}=p \frac{C_{1}}{P_{1}}+(1-p) \frac{C_{2}}{P_{2}} .
$$

- Above,

$$
p \equiv \frac{(S / P)-\left(S_{2} / P_{2}\right)}{\left(S_{1} / P_{1}\right)-\left(S_{2} / P_{2}\right)} .
$$

- The derivative's price using asset two as numeraire is thus a martingale under the risk-neutral probability $p$

- The expected returns of the two assets are irrelevant.

\section{Brownian Motion ${ }^{\mathrm{a}}$}

- Brownian motion is a stochastic process $\{X(t), t \geq 0\}$ with the following properties.

1. $X(0)=0$, unless stated otherwise.

2. for any $0 \leq t_{0}<t_{1}<\cdots<t_{n}$, the random variables

$$
X\left(t_{k}\right)-X\left(t_{k-1}\right)
$$

for $1 \leq k \leq n$ are independent. ${ }^{\mathrm{b}}$

3. for $0 \leq s<t, X(t)-X(s)$ is normally distributed with mean $\mu(t-s)$ and variance $\sigma^{2}(t-s)$, where $\mu$ and $\sigma \neq 0$ are real numbers.

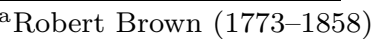

${ }^{\mathrm{b}}$ So $X(t)-X(s)$ is independent of $X(r)$ for $r \leq s<t$. 


\section{Brownian Motion (concluded)}

- Such a process will be called a $(\mu, \sigma)$ Brownian motion with drift $\mu$ and variance $\sigma^{2}$.

- The existence and uniqueness of such a process is guaranteed by Wiener's theorem. ${ }^{\text {a }}$

- Although Brownian motion is a continuous function of $t$ with probability one, it is almost nowhere differentiable.

- The $(0,1)$ Brownian motion is also called the Wiener process.

${ }^{\mathrm{a} N o r b e r t ~ W i e n e r ~(1894-1964) . ~}$

\section{Brownian Motion Is a Random Walk in Continuous} Time

Claim $1 A(\mu, \sigma)$ Brownian motion is the limiting case of random walk.

- A particle moves $\Delta x$ to the left with probability $1-p$.

- It moves to the right with probability $p$ after $\Delta t$ time.

- Assume $n \equiv t / \Delta t$ is an integer.

- Its position at time $t$ is

$$
Y(t) \equiv \Delta x\left(X_{1}+X_{2}+\cdots+X_{n}\right)
$$

\section{Brownian Motion as Limit of Random Walk} (continued)

\section{Example}

- If $\{X(t), t \geq 0\}$ is the Wiener process, then $X(t)-X(s) \sim N(0, t-s)$.

- A $(\mu, \sigma)$ Brownian motion $Y=\{Y(t), t \geq 0\}$ can be expressed in terms of the Wiener process:

$$
Y(t)=\mu t+\sigma X(t)
$$

- Note that $Y(t+s)-Y(t) \sim N\left(\mu s, \sigma^{2} s\right)$.
- (continued)

- Here

$$
X_{i} \equiv \begin{cases}+1 & \text { if the } i \text { th move is to the right } \\ -1 & \text { if the } i \text { th move is to the left. }\end{cases}
$$

- $X_{i}$ are independent with

$$
\operatorname{Prob}\left[X_{i}=1\right]=p=1-\operatorname{Prob}\left[X_{i}=-1\right] \text {. }
$$

- Recall $E\left[X_{i}\right]=2 p-1$ and $\operatorname{Var}\left[X_{i}\right]=1-(2 p-1)^{2}$. 
Brownian Motion as Limit of Random Walk (continued)

- Therefore,

$$
\begin{aligned}
E[Y(t)] & =n(\Delta x)(2 p-1), \\
\operatorname{Var}[Y(t)] & =n(\Delta x)^{2}\left[1-(2 p-1)^{2}\right] .
\end{aligned}
$$

- With $\Delta x \equiv \sigma \sqrt{\Delta t}$ and $p \equiv[1+(\mu / \sigma) \sqrt{\Delta t}] / 2$,

$$
\begin{aligned}
E[Y(t)] & =n \sigma \sqrt{\Delta t}(\mu / \sigma) \sqrt{\Delta t}=\mu t, \\
\operatorname{Var}[Y(t)] & =n \sigma^{2} \Delta t\left[1-(\mu / \sigma)^{2} \Delta t\right] \rightarrow \sigma^{2} t
\end{aligned}
$$

as $\Delta t \rightarrow 0$.

\section{Geometric Brownian Motion}

- Let $X \equiv\{X(t), t \geq 0\}$ be a Brownian motion process.

- The process

$$
\left\{Y(t) \equiv e^{X(t)}, t \geq 0\right\},
$$

is called geometric Brownian motion.

- Suppose further that $X$ is a $(\mu, \sigma)$ Brownian motion.

- $X(t) \sim N\left(\mu t, \sigma^{2} t\right)$ with moment generating function

$$
E\left[e^{s X(t)}\right]=E\left[Y(t)^{s}\right]=e^{\mu t s+\left(\sigma^{2} t s^{2} / 2\right)}
$$

from Eq. (16) on p 139.

\section{Geometric Brownian Motion (continued)}

- In particular,

$$
\begin{aligned}
E[Y(t)] & =e^{\mu t+\left(\sigma^{2} t / 2\right)} \\
\operatorname{Var}[Y(t)] & =E\left[Y(t)^{2}\right]-E[Y(t)]^{2} \\
& =e^{2 \mu t+\sigma^{2} t}\left(e^{\sigma^{2} t}-1\right) .
\end{aligned}
$$

- Similarity to the the BOPM: The $p$ is identical to the probability in Eq. (23) on p. 235 and $\Delta x=\ln u$. 


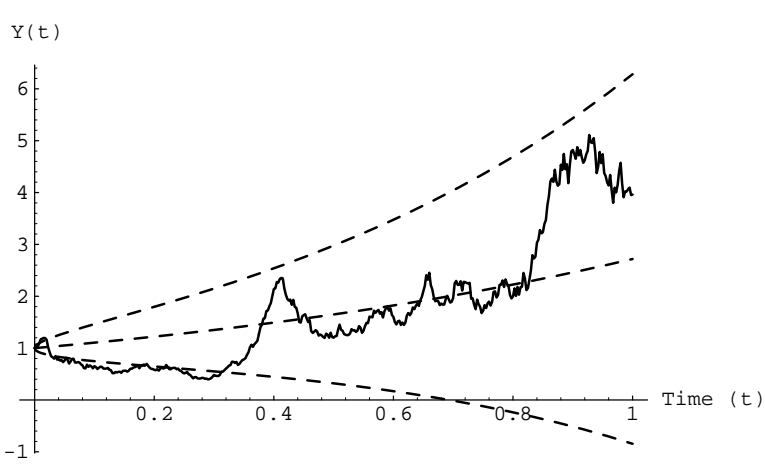

\section{Geometric Brownian Motion (concluded)}

- Then

$$
\ln Y_{n}=\sum_{i=1}^{n} \ln X_{i}
$$

is a sum of independent, identically distributed random variables.

- Thus $\left\{\ln Y_{n}, n \geq 0\right\}$ is approximately Brownian motion.

- And $\left\{Y_{n}, n \geq 0\right\}$ is approximately geometric Brownian motion.

\section{Geometric Brownian Motion (continued)}

- It is useful for situations in which percentage changes are independent and identically distributed.

- Let $Y_{n}$ denote the stock price at time $n$ and $Y_{0}=1$.

- Assume relative returns

$$
X_{i} \equiv \frac{Y_{i}}{Y_{i-1}}
$$

are independent and identically distributed.

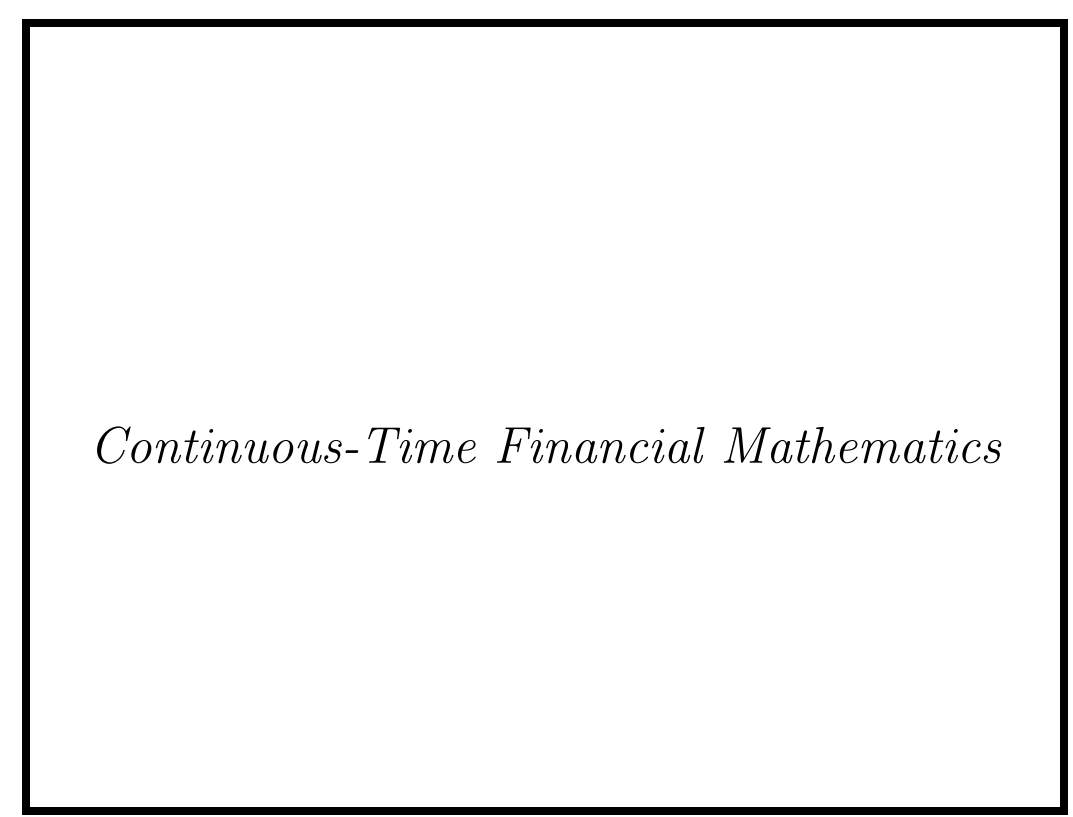


A proof is that which convinces a reasonable man; a rigorous proof is that which convinces an unreasonable man - Mark Kac (1914-1984)

The pursuit of mathematics is a divine madness of the human spirit. - Alfred North Whitehead (1861-1947),

Science and the Modern World

\section{Stochastic Integrals (concluded)}

- Typical requirements for $X$ in financial applications are:

$-\operatorname{Prob}\left[\int_{0}^{t} X^{2}(s) d s<\infty\right]=1$ for all $t \geq 0$ or the stronger $\int_{0}^{t} E\left[X^{2}(s)\right] d s<\infty$.

- The information set at time $t$ includes the history of $X$ and $W$ up to that point in time.

- But it contains nothing about the evolution of $X$ or $W$ after $t$ (nonanticipating, so to speak).

- The future cannot influence the present.

- $\{X(s), 0 \leq s \leq t\}$ is independent of $\{W(t+u)-W(t), u>0\}$

\section{Stochastic Integrals}

- Use $W \equiv\{W(t), t \geq 0\}$ to denote the Wiener process.

- The goal is to develop integrals of $X$ from a class of stochastic processes, ${ }^{\mathrm{a}}$

$$
\mathrm{I}_{t}(X) \equiv \int_{0}^{t} X d W, \quad t \geq 0 .
$$

- $\mathrm{I}_{t}(X)$ is a random variable called the stochastic integral of $X$ with respect to $W$.

- The stochastic process $\left\{\mathrm{I}_{t}(X), t \geq 0\right\}$ will be denoted by $\int X d W$.

- A stochastic process $\{X(t)\}$ is simple if there exist $0=t_{0}<t_{1}<\cdots$ such that

$$
X(t)=X\left(t_{k-1}\right) \text { for } t \in\left[t_{k-1}, t_{k}\right), k=1,2, \ldots
$$

for any realization (see figure next page). 


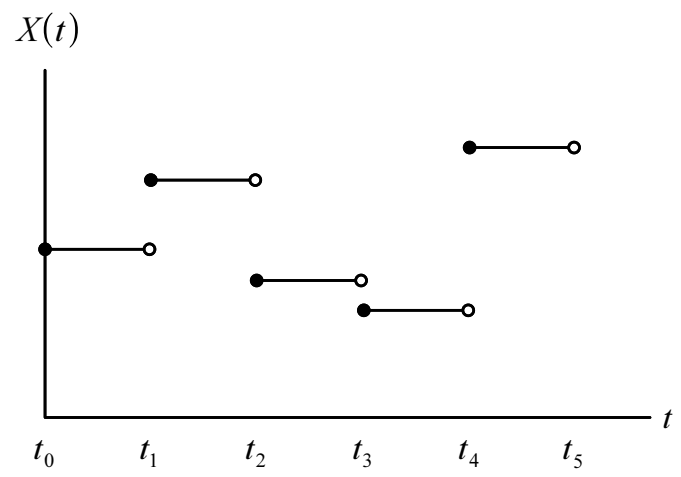

\section{Ito Integral (continued)}

- Let $X=\{X(t), t \geq 0\}$ be a general stochastic process.

- Then there exists a random variable $\mathrm{I}_{t}(X)$, unique almost certainly, such that $\mathrm{I}_{t}\left(X_{n}\right)$ converges in probability to $\mathrm{I}_{t}(X)$ for each sequence of simple stochastic processes $X_{1}, X_{2}, \ldots$ such that $X_{n}$ converges in probability to $X$.

- If $X$ is continuous with probability one, then $\mathrm{I}_{t}\left(X_{n}\right)$ converges in probability to $\mathrm{I}_{t}(X)$ as $\delta_{n} \equiv \max _{1 \leq k \leq n}\left(t_{k}-t_{k-1}\right)$ goes to zero.

\section{Ito Integral (continued)}

- The Ito integral of a simple process is defined as

$$
\mathrm{I}_{t}(X) \equiv \sum_{k=0}^{n-1} X\left(t_{k}\right)\left[W\left(t_{k+1}\right)-W\left(t_{k}\right)\right],
$$

where $t_{n}=t$

- The integrand $X$ is evaluated at $t_{k}$, not $t_{k+1}$.

- Define the Ito integral of more general processes as a limiting random variable of the Ito integral of simple stochastic processes.

\section{Ito Integral (concluded)}

- It is a fundamental fact that $\int X d W$ is continuous almost surely.

- The following theorem says the Ito integral is a martingale.

- A corollary is the mean value formula

$$
E\left[\int_{a}^{b} X d W\right]=0 .
$$

Theorem 15 The Ito integral $\int X d W$ is a martingale. 


\section{Discrete Approximation}

- Recall Eq. (46) on p. 445.

- The following simple stochastic process $\{\widehat{X}(t)\}$ can be used in place of $X$ to approximate the stochastic integral $\int_{0}^{t} X d W$

$$
\widehat{X}(s) \equiv X\left(t_{k-1}\right) \text { for } s \in\left[t_{k-1}, t_{k}\right), k=1,2, \ldots, n .
$$

- Note the nonanticipating feature of $\widehat{X}$.

- The information up to time $s$,

$$
\{\widehat{X}(t), W(t), 0 \leq t \leq s\},
$$

cannot determine the future evolution of $X$ or $W$.

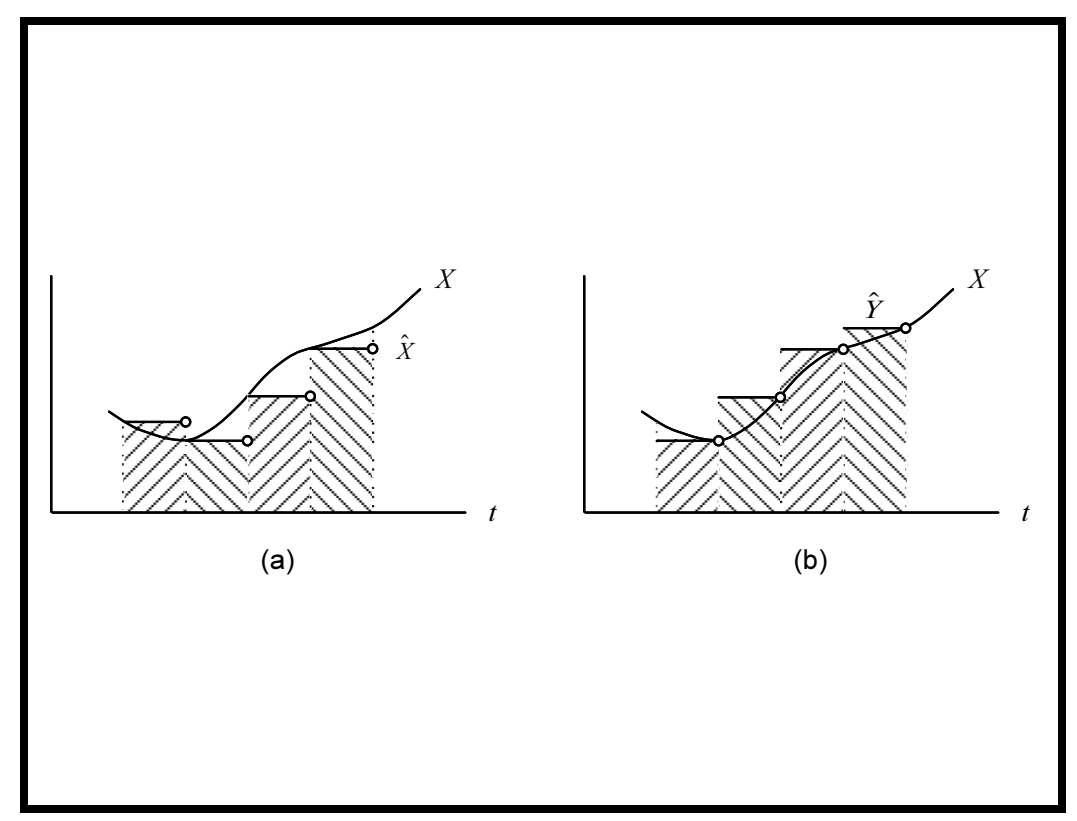

\section{Discrete Approximation (concluded)}

- Suppose we defined the stochastic integral as

$$
\sum_{k=0}^{n-1} X\left(t_{k+1}\right)\left[W\left(t_{k+1}\right)-W\left(t_{k}\right)\right] .
$$

- Then we would be using the following different simple stochastic process in the approximation,

$$
\widehat{Y}(s) \equiv X\left(t_{k}\right) \text { for } s \in\left[t_{k-1}, t_{k}\right), k=1,2, \ldots, n .
$$

- This clearly anticipates the future evolution of $X$. 


\section{Ito Process (continued)}

- A shorthand $\mathrm{d}^{\mathrm{a}}$ is the following stochastic differential equation for the Ito differential $d X_{t}$,

$$
d X_{t}=a\left(X_{t}, t\right) d t+b\left(X_{t}, t\right) d W_{t}
$$

- Or simply $d X_{t}=a_{t} d t+b_{t} d W_{t}$.

- This is Brownian motion with an instantaneous drift $a_{t}$ and an instantaneous variance $b_{t}^{2}$.

- $X$ is a martingale if the drift $a_{t}$ is zero by Theorem 15 (p. 447).

${ }^{a}$ Paul Langevin (1904).

\section{Euler Approximation}

- The following approximation follows from Eq. (48),

$$
\begin{aligned}
& \widehat{X}\left(t_{n+1}\right) \\
= & \widehat{X}\left(t_{n}\right)+a\left(\widehat{X}\left(t_{n}\right), t_{n}\right) \Delta t+b\left(\widehat{X}\left(t_{n}\right), t_{n}\right) \Delta W\left(t_{n}\right),
\end{aligned}
$$

where $t_{n} \equiv n \Delta t$.

- It is called the Euler or Euler-Maruyama method.

- Under mild conditions, $\widehat{X}\left(t_{n}\right)$ converges to $X\left(t_{n}\right)$.

- Recall that $\Delta W\left(t_{n}\right)$ should be interpreted as $W\left(t_{n+1}\right)-W\left(t_{n}\right)$ instead of $W\left(t_{n}\right)-W\left(t_{n-1}\right)$.

\section{Ito Process (concluded)}

- $d W$ is normally distributed with mean zero and variance $d t$

- An equivalent form to Eq. (47) is

$$
d X_{t}=a_{t} d t+b_{t} \sqrt{d t} \xi
$$

where $\xi \sim N(0,1)$.

- This formulation makes it easy to derive Monte Carlo simulation algorithms.

\section{More Discrete Approximations}

- Under fairly loose regularity conditions, approximation (49) on p. 454 can be replaced by

$$
\begin{aligned}
& \widehat{X}\left(t_{n+1}\right) \\
= & \widehat{X}\left(t_{n}\right)+a\left(\widehat{X}\left(t_{n}\right), t_{n}\right) \Delta t+b\left(\widehat{X}\left(t_{n}\right), t_{n}\right) \sqrt{\Delta t} Y\left(t_{n}\right) .
\end{aligned}
$$

- $Y\left(t_{0}\right), Y\left(t_{1}\right), \ldots$ are independent and identically distributed with zero mean and unit variance. 


\section{More Discrete Approximations (concluded)}

- A simpler discrete approximation scheme:

$$
\begin{aligned}
& \widehat{X}\left(t_{n+1}\right) \\
= & \widehat{X}\left(t_{n}\right)+a\left(\widehat{X}\left(t_{n}\right), t_{n}\right) \Delta t+b\left(\widehat{X}\left(t_{n}\right), t_{n}\right) \sqrt{\Delta t} \xi .
\end{aligned}
$$

$-\operatorname{Prob}[\xi=1]=\operatorname{Prob}[\xi=-1]=1 / 2$.

- Note that $E[\xi]=0$ and $\operatorname{Var}[\xi]=1$.

- This clearly defines a binomial model.

- As $\Delta t$ goes to zero, $\widehat{X}$ converges to $X$.

\section{Trading and the Ito Integral (concluded)}

- The equivalent Ito integral,

$$
G_{T}(\phi) \equiv \int_{0}^{T} \phi_{t} d \boldsymbol{S}_{t}=\int_{0}^{T} \phi_{t} \mu_{t} d t+\int_{0}^{T} \phi_{t} \sigma_{t} d W_{t}
$$

measures the gains realized by the trading strategy over the period $[0, T]$.

\section{Ito's Lemma}

A smooth function of an Ito process is itself an Ito process.

Theorem 16 Suppose $f: R \rightarrow R$ is twice continuously differentiable and $d X=a_{t} d t+b_{t} d W$. Then $f(X)$ is the Ito process,

$$
\begin{aligned}
& f\left(X_{t}\right) \\
= & f\left(X_{0}\right)+\int_{0}^{t} f^{\prime}\left(X_{s}\right) a_{s} d s+\int_{0}^{t} f^{\prime}\left(X_{s}\right) b_{s} d W \\
+ & \frac{1}{2} \int_{0}^{t} f^{\prime \prime}\left(X_{s}\right) b_{s}^{2} d s
\end{aligned}
$$

for $t \geq 0$. 


\section{Ito's Lemma (continued)}

- In differential form, Ito's lemma becomes

$$
d f(X)=f^{\prime}(X) a d t+f^{\prime}(X) b d W+\frac{1}{2} f^{\prime \prime}(X) b^{2} d t
$$

- Compared with calculus, the interesting part is the third term on the right-hand side.

- A convenient formulation of Ito's lemma is

$$
d f(X)=f^{\prime}(X) d X+\frac{1}{2} f^{\prime \prime}(X)(d X)^{2} .
$$

\section{Ito's Lemma (continued)}

Theorem 17 (Higher-Dimensional Ito's Lemma) Let $W_{1}, W_{2}, \ldots, W_{n}$ be independent Wiener processes and $X \equiv\left(X_{1}, X_{2}, \ldots, X_{m}\right)$ be a vector process. Suppose $f: R^{m} \rightarrow R$ is twice continuously differentiable and $X_{i}$ is an Ito process with $d X_{i}=a_{i} d t+\sum_{j=1}^{n} b_{i j} d W_{j}$. Then $d f(X)$ is an Ito process with the differential,

$$
d f(X)=\sum_{i=1}^{m} f_{i}(X) d X_{i}+\frac{1}{2} \sum_{i=1}^{m} \sum_{k=1}^{m} f_{i k}(X) d X_{i} d X_{k}
$$

where $f_{i} \equiv \partial f / \partial x_{i}$ and $f_{i k} \equiv \partial^{2} f / \partial x_{i} \partial x_{k}$.

\section{Ito's Lemma (continued)}

- We are supposed to multiply out $(d X)^{2}=(a d t+b d W)^{2}$ symbolically according to

\begin{tabular}{|c|cc|}
\hline$\times$ & $d W$ & $d t$ \\
\hline$d W$ & $d t$ & 0 \\
$d t$ & 0 & 0 \\
\hline
\end{tabular}

- The $(d W)^{2}=d t$ entry is justified by a known result.

- This form is easy to remember because of its similarity to the Taylor expansion.

\section{Ito's Lemma (continued)}

- The multiplication table for Theorem 17 is

\begin{tabular}{|c|cc|}
\hline$\times$ & $d W_{i}$ & $d t$ \\
\hline$d W_{k}$ & $\delta_{i k} d t$ & 0 \\
$d t$ & 0 & 0 \\
\hline
\end{tabular}

in which

$$
\delta_{i k}= \begin{cases}1 & \text { if } i=k \\ 0 & \text { otherwise }\end{cases}
$$




\section{Ito's Lemma (continued)}

Theorem 18 (Alternative Ito's Lemma) Let $W_{1}, W_{2}, \ldots, W_{m}$ be Wiener processes and $X \equiv\left(X_{1}, X_{2}, \ldots, X_{m}\right)$ be a vector process. Suppose $f: R^{m} \rightarrow R$ is twice continuously differentiable and $X_{i}$ is an Ito process with $d X_{i}=a_{i} d t+b_{i} d W_{i}$. Then $d f(X)$ is the following Ito process,

$$
d f(X)=\sum_{i=1}^{m} f_{i}(X) d X_{i}+\frac{1}{2} \sum_{i=1}^{m} \sum_{k=1}^{m} f_{i k}(X) d X_{i} d X_{k} .
$$

\section{Geometric Brownian Motion}

- Consider the geometric Brownian motion process $Y(t) \equiv e^{X(t)}$

- $X(t)$ is a $(\mu, \sigma)$ Brownian motion.

- Hence $d X=\mu d t+\sigma d W$ by Eq. (45) on p. 429.

- As $\partial Y / \partial X=Y$ and $\partial^{2} Y / \partial X^{2}=Y$, Ito's formula (50) on p. 460 implies

$$
\begin{aligned}
d Y & =Y d X+(1 / 2) Y(d X)^{2} \\
& =Y(\mu d t+\sigma d W)+(1 / 2) Y(\mu d t+\sigma d W)^{2} \\
& =Y(\mu d t+\sigma d W)+(1 / 2) Y \sigma^{2} d t .
\end{aligned}
$$

\section{Ito's Lemma (concluded)}

- The multiplication table for Theorem 18 is

\begin{tabular}{|c|cc|}
\hline$\times$ & $d W_{i}$ & $d t$ \\
\hline$d W_{k}$ & $\rho_{i k} d t$ & 0 \\
$d t$ & 0 & 0 \\
\hline
\end{tabular}

- Here, $\rho_{i k}$ denotes the correlation between $d W_{i}$ and $d W_{k}$.

\section{Geometric Brownian Motion (concluded)}

- Hence

$$
\frac{d Y}{Y}=\left(\mu+\sigma^{2} / 2\right) d t+\sigma d W
$$

- The annualized instantaneous rate of return is $\mu+\sigma^{2} / 2$ not $\mu$. 


\section{Product of Geometric Brownian Motion Processes}

- Let

$$
\begin{aligned}
d Y / Y & =a d t+b d W_{Y}, \\
d Z / Z & =f d t+g d W_{Z} .
\end{aligned}
$$

- Consider the Ito process $U \equiv Y Z$.

- Apply Ito's lemma (Theorem 18 on p. 464):

$$
\begin{aligned}
d U= & Z d Y+Y d Z+d Y d Z \\
= & Z Y\left(a d t+b d W_{Y}\right)+Y Z\left(f d t+g d W_{Z}\right) \\
& +Y Z\left(a d t+b d W_{Y}\right)\left(f d t+g d W_{Z}\right) \\
= & U(a+f+b g \rho) d t+U b d W_{Y}+U g d W_{Z} .
\end{aligned}
$$

Product of Geometric Brownian Motion Processes (concluded)

- $\ln U$ is Brownian motion with a mean equal to the sum of the means of $\ln Y$ and $\ln Z$.

- This holds even if $Y$ and $Z$ are correlated.

- Finally, $\ln Y$ and $\ln Z$ have correlation $\rho$.
Product of Geometric Brownian Motion Processes (continued)

- The product of two (or more) correlated geometric Brownian motion processes thus remains geometric Brownian motion.

- Note that

$$
\begin{aligned}
Y & =\exp \left[\left(a-b^{2} / 2\right) d t+b d W_{Y}\right] \\
Z & =\exp \left[\left(f-g^{2} / 2\right) d t+g d W_{Z}\right] \\
U & =\exp \left[\left(a+f-\left(b^{2}+g^{2}\right) / 2\right) d t+b d W_{Y}+g d W_{Z}\right]
\end{aligned}
$$

- Suppose $Y$ and $Z$ are drawn from p. 468.

- Let $U \equiv Y / Z$.

- We now show that

$$
\frac{d U}{U}=\left(a-f+g^{2}-b g \rho\right) d t+b d W_{Y}-g d W_{Z}
$$

- Keep in mind that $d W_{Y}$ and $d W_{Z}$ have correlation $\rho$. 


\section{Quotients of Geometric Brownian Motion Processes} (concluded)

- The multidimensional Ito's lemma (Theorem 18 on p. 464) can be employed to show that

$$
d U
$$

$=(1 / Z) d Y-\left(Y / Z^{2}\right) d Z-\left(1 / Z^{2}\right) d Y d Z+\left(Y / Z^{3}\right)(d Z)^{2}$

$=(1 / Z)\left(a Y d t+b Y d W_{Y}\right)-\left(Y / Z^{2}\right)\left(f Z d t+g Z d W_{Z}\right)$ $-\left(1 / Z^{2}\right)(b g Y Z \rho d t)+\left(Y / Z^{3}\right)\left(g^{2} Z^{2} d t\right)$

$=U\left(a d t+b d W_{Y}\right)-U\left(f d t+g d W_{Z}\right)$ $-U(b g \rho d t)+U\left(g^{2} d t\right)$

$=U\left(a-f+g^{2}-b g \rho\right) d t+U b d W_{Y}-U g d W_{Z}$. 